\title{
Adaptive Detection of Terminal Voltage Collapses for Li-Ion Batteries
}

\author{
Shayok Mukhopadhyay and Fumin Zhang
}

\begin{abstract}
We introduce a novel approach for detecting and predicting terminal voltage collapses in Li-ion batteries without having complete knowledge of a battery model. We present a simplified dynamic model for a Li-ion battery that is forced to track the output voltage curve of a physical Liion battery by using universal adaptive stabilization (UAS). We prove that when the physical Li-ion battery becomes unstable, then the simplified dynamic model becomes unstable. Our results do not require a sophisticated model for a battery that faithfully captures all dynamics. Using our results, we present an algorithm for detecting impending voltage collapses for $\mathrm{Li}$ ion batteries.
\end{abstract}

\section{INTRODUCTION}

Low self-discharge rate, no memory effect and high energy density are some of the characteristics [1] that make Li-ion batteries viable as power sources for various applications. This has motivated some recent work related to batteries [2][4]. The terminal voltage of a battery drops sharply from its operating value when it is in a low state of charge $(\mathrm{SoC})$. Systems like autonomous vehicles can have multiple batteries on board. If it is detected that the terminal voltage of a particular battery is about to collapse, appropriate actions can be taken to switch to a backup battery.

The terminal voltage, available capacity, state of charge (SoC) and state of health $(\mathrm{SoH})$ [5]-[8] can determine if the terminal voltage of a battery is going to collapse. Usually a constant threshold voltage is used to determine that a particular battery is discharged [6], [7]. The terminal voltage of a battery depends on the discharge current it supplies. So using a constant voltage threshold can lead to false alarms in the presence of noise or large spikes in the discharge current. Another strategy is to use a threshold on the battery state of charge (SoC). This strategy is affected by load demand, number of charge-discharge cycles and temperature. Determining the SoC involves coulomb counting, which introduces errors as the measured input current is integrated in the presence of measurement errors [6].

Incorporating battery models improves the accuracy of detecting an impending terminal voltage collapse. Various types of battery models and associated identification techniques exist [8]-[14]. Dynamic battery models along with adaptive thresholds [15] can overcome some problems with constant thresholds. Filtering algorithms [16] for state estimation and fault detection strategies like residual generation [17] can

This research was partially supported by the ONR grants N00014-081-1007, N00014-09-1-1074, and N00014-10-10712 (YIP), and NSF grants ECCS-0841195 (CAREER), CNS-0931576, and ECCS-1056253. Shayok Mukhopadhyay and Fumin Zhang are with the School of Electrical and Computer Engineering, Georgia Institute of Technology, Atlanta, GA 30322, USA. Emails:\{shayok, fumin\}@gatech.edu also be used. All the above methods require detailed battery models. Substantial time and effort [8] is required to obtain such models. Also in reality battery characteristics may differ from the model used; resulting in voltage collapse before a particular algorithm detects it. Existing results are either dependent on a particular testing methodology or on a type of model [6]-[8], [16], [17]. Our work aims to reduce such dependence without sacrificing the ability to detect voltage collapses.

The contributions of this paper are as follows. We present a general method for detecting Li-ion battery voltage collapses without the requirement of a detailed model. Our method only requires the measurement of the terminal voltage of a battery and works in the presence of measurement noise or voltage spikes due to non-smooth current discharges. We do not measure the discharge current and do not use coulomb counting techniques. Thus the cost of accurate current measurement and associated errors are eliminated. We do not estimate the SoC of a battery and do not need a static threshold on the $\mathrm{SoC}$ or terminal voltage, hence our method is robust to variations in the $\mathrm{SoC}$ and the terminal voltage.

Our method is motivated by the following observation. The terminal voltage curve vs. time for any battery is linear for most of its usable life in a charge-discharge cycle. Towards the end of its life (i.e. low SoC), the terminal voltage starts declining. When the $\mathrm{SoC}$ is low the decline is slow (but not linear) initially. Further decrease in SoC causes near instantaneous decline. Our method aims at detecting the initial slow decline of terminal voltage when the $\mathrm{SoC}$ is low. To achieve our goal we construct a simplified dynamic model mimicking the behavior of a Li-ion battery and force it to track the output of a physical battery using UAS [18], [19]. From the states of our simplified model we can decide if the terminal voltage of the physical battery has entered the region of slow, nonlinear decline. In the remainder of the paper, we first present some background information on battery models and UAS. Then we present our results and an algorithm to detect terminal voltage collapses for Li-ion batteries and some simulations.

\section{BACKGROUND}

\section{A. Chen and Mora's (CM) battery model}

Figure 1 is an equivalent circuit representation of a $\mathrm{Li}$ ion battery [9] showing two coupled circuits. The left half models the variation of the state of charge $(\mathrm{SoC}) x_{1}$ and the right half models the variation of battery output voltage $y$ as a function of the charge/discharge current $i(t)$. All the 


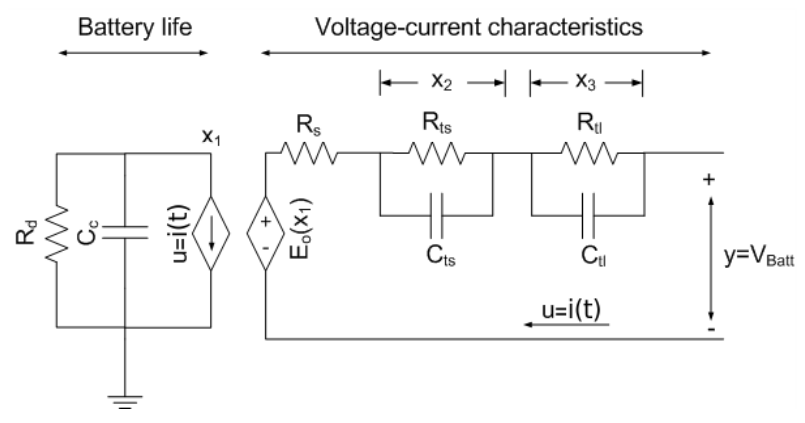

Fig. 1. Chen and Mora's battery model

circuit components $C_{t s}, C_{t l}, R_{s}, R_{t s}, R_{t l}, E_{o}$ are functions of $x_{1}$ represented as follows:

$$
\begin{aligned}
C_{t s} & =-k_{4} e^{-k_{1} x_{1}}+k_{3} \\
C_{t l} & =-k_{6} e^{-k_{2} x_{1}}+k_{5} \\
R_{s} & =k_{7} e^{-k_{8} x_{1}}+k_{9} \\
R_{t s} & =k_{10} e^{-k_{11} x_{1}}+k_{12} \\
R_{t l} & =k_{13} e^{-k_{14} x_{1}}+k_{15} \\
E_{o} & =-k_{16} e^{-k_{17} x_{1}}+k_{18}+k_{19} x_{1} \\
& -k_{20} x_{1}^{2}+k_{21} x_{1}^{3} \\
C_{c} & =3600 C f_{1} f_{2} .
\end{aligned}
$$

where $k_{i}>0$ for $i=1,2, \ldots, 21$ and $k_{1}<k_{2}<k_{3}<$ $k_{4}<k_{5}<k_{6}$. In eqn. (7) $f_{1}, f_{2} \in[0,1]$ are factors taking into account the effects of temperature and charge-discharge cycles respectively. By default, $f_{1}=f_{2}=1$, but their values will decrease after each charge-discharge cycle. $E_{o}$ is the open-circuit voltage of the battery. In general it can be said that $E_{o}:[0,1] \rightarrow\left[\Omega_{1}, \Omega_{2}\right]$ where $\Omega_{1}, \Omega_{2} \in \mathbb{R}$ and $\Omega_{2}>$ $\Omega_{1}>0$. The various resistances, capacitances, and constants $\left(k_{1}, \cdots, k_{21}\right)$ shown here are independent of $i(t)$.

Knauff et.al. [12] provide the following state space realization of the battery model.

$$
\begin{aligned}
\dot{x}_{1} & =-\frac{1}{C_{c}} i \\
\dot{x}_{2} & =-\frac{x_{2}}{R_{t s} C_{t s}}+\frac{i}{C_{t s}} \\
\dot{x}_{3} & =-\frac{x_{3}}{R_{t l} C_{t l}}+\frac{i}{C_{t l}} \\
y & =E_{o}-x_{2}-x_{3}-i R_{s},
\end{aligned}
$$

where $y$ represents the voltage output from the battery, $x_{2}$ represents the voltage drop across $R_{t s} \| C_{t s}$ and $x_{3}$ represents the voltage drop across $R_{t l} \| C_{t l} . x_{1} \in[0,1], x_{2}, x_{3} \in \mathbb{R}^{+}$ with initial conditions $\mathbf{x}_{0}=[1,0,0]^{\top}$.

\section{B. Universal adaptive stabilization (UAS)}

The details concerning UAS can be found in [18]. We use UAS with a particular class of switching functions known as Nussbaum functions [18]. It is shown in [20] and [21] that $E_{\alpha}\left(-\lambda t^{\alpha}\right)$ is a Nussbaum function for $\lambda>0$ and $\alpha \in$ $(2,3]$ where $E_{\alpha}(z)$ is the Mittag-Leffler (ML) function in one parameter given by

$$
E_{\alpha}(z)=\sum_{k=0}^{\infty} \frac{z^{k}}{\Gamma(k \alpha+1)},
$$

Figure 2 shows what the Nussbaum function with MittagLeffler form looks like. It produces growing oscillations over time. We use a Nussbaum function with ML form in this paper.

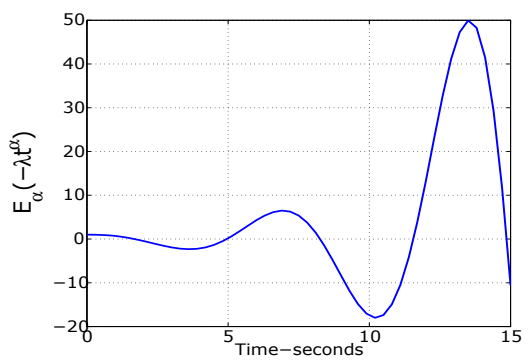

Fig. 2. The Mittag-Leffler (ML) function as a Nussbaum function

\section{CONFIGURATION FOR DETECTING BATTERY TERMINAL VOLTAGE COLLAPSES USING UAS}

The CM model shown in section II-A is fairly accurate compared to experimental data. The process of obtaining eqns. (1)-(7) takes considerable experimental effort [8], [9], [13], [14] and the various constants $k_{1}-k_{21}$ may be different for each individual Li-ion battery. We propose the following simplified model having characteristics similar to the CM model.

$$
\begin{aligned}
\dot{\hat{\rho}} & =-\frac{1}{\widehat{C}_{c}} u \\
\dot{\widehat{x}}_{1} & =-c_{1} \widehat{x}_{1}+\frac{c_{3}}{\widehat{\rho}}+u, c_{1}>0, c_{3}=1 \\
\dot{\hat{x}}_{2} & =-c_{2} \widehat{x}_{2}+\frac{c_{4}}{\widehat{\rho}}+u, c_{2}>0, c_{4}=1 \\
\widehat{y} & =\widehat{y}_{0}+m \widehat{\rho}-\widehat{x}_{1}-\widehat{x}_{2}
\end{aligned}
$$

where $\widehat{\rho} \in[0,1], u, \widehat{y} \in\left[\widehat{y}_{0}, 0\right]$ represent the SoC, discharge current and the output terminal voltage for this model. $\widehat{C}_{c}$ represents the Ampere-hour (Ah) capacity of the simplified battery model. $\widehat{C}_{c}$ should equal the Ah capacity of the battery we plan to model using eqns. (13)-(16). States $\widehat{x}_{1}, \widehat{x}_{2} \in \mathbb{R}$ represent transient voltages. Let $\widehat{\rho}_{0}=1, \widehat{\mathbf{x}}_{0}=[0,0]^{\top}$, $\widehat{y}=\widehat{y}_{0}$ be the initial values for the SoC, the states and the output respectively. The terminal voltage for a new battery on no load is represented by $\widehat{y}_{0}$. The term $\widehat{y}_{0}+m \widehat{\rho}, m>0$ decreases linearly with $\widehat{\rho}$ mimicking the linear decline of battery terminal voltage. Assuming that $u$ is bounded, $\dot{\widehat{x}}_{1}$ and $\dot{\widehat{x}}_{2}$ tend to infinity as $\widehat{\rho} \rightarrow 0$. This causes $\widehat{x}_{1}$ and $\widehat{x}_{2}$ to tend to infinity as $\widehat{\rho} \rightarrow 0$, thus causing the output voltage $\widehat{y} \rightarrow 0$. This mimics the drop in terminal voltage as the $\mathrm{SoC}$ of a battery goes to zero. From eqn. (16) we observe that if the output $\widehat{y}$ is forced to follow the output $y$ of a physical battery (sufficiently close) and $y$ decreases suddenly then the states $\widehat{x}_{1}$ and $\widehat{x}_{2}$ must increase. We thus hypothesize that the actual Li-ion battery is unstable when the simplified battery model 


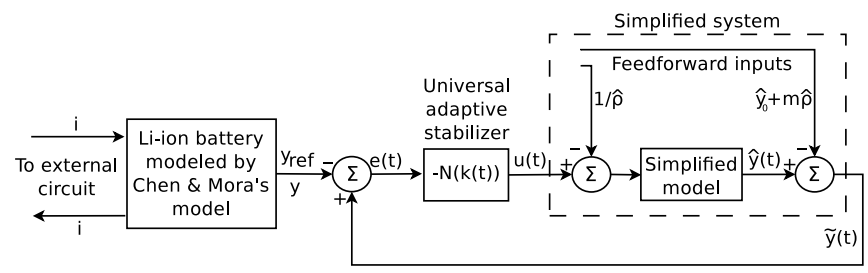

Fig. 3. Battery output voltage tracking with UAS

is unstable and if this instability can be detected then the terminal voltage collapse of a Li-ion battery can be detected.

Figure 3 shows the setup we propose to detect the terminal voltage collapse of a Li-ion battery, it consists of three main blocks. The first block to the left represents the real Li-ion battery (which we assume is modeled sufficiently accurately using the CM model). The block in the middle represents the universal adaptive stabilizer. The block shown using a dotted rectangle is the simplified system formed by adding feedforward inputs to the simplified model shown in eqns. (14)-(16). The simplified system is obtained as,

$$
\begin{aligned}
\dot{\widehat{x}}_{1} & =-c_{1} \widehat{x}_{1}+u, c_{1}>0 \\
\dot{\widehat{x}}_{2} & =-c_{2} \widehat{x}_{2}+u, c_{2}>0 \\
\widetilde{y} & =-\widehat{x}_{1}-\widehat{x}_{2} .
\end{aligned}
$$

\section{TERMINAL VOLTAGE COLLAPSE DETECTION ALGORITHM}

In this section we analyze the closed loop dynamics of the system shown in fig. 3. Finally we provide an algorithm to detect terminal voltage collapses for Li-ion batteries and theoretically justify the result.

\section{A. Closed loop dynamics}

The closed loop shown in fig. 3 consists of the simplified system connected to the universal adaptive stabilizer via the following feedback law.

$$
\left.\begin{array}{rl}
u(t) & =-N(k(t)) e(t) \\
N(k(t)) & =E_{\alpha}\left(-\lambda k(t)^{\alpha}\right), \lambda>0, \alpha \in(2,3) \\
\dot{k}(t) & =e^{2}(t) \\
e(t) & =\widetilde{y}(t)-y(t)
\end{array}\right\}
$$

We will now show that the above law will drive the error $e(t)$ to zero as time goes to infinity.

Lemma 4.1: Let $y$ be the output of a real battery described by the CM model. Let $u, \widetilde{y}$ be the input to, and output from the simplified system in (17)-(19) respectively. Let $d(t)=$ $\left(c_{2}-c_{1}\right) \widehat{x}_{2}-c_{1} y(t)-\dot{y}(t)$. Assuming $\left(\int_{0}^{\infty}|d(t)|^{p} d t\right)^{\frac{1}{p}}<$ $\infty$, if the feedback law $u(t)=-N(k(t)) e(t), \dot{k}(t)=$ $e^{2}(t)$ is used with the closed loop system in Fig. 3 then $\lim _{t \rightarrow \infty} k(t)=k_{\infty}$ and $\lim _{t \rightarrow \infty} e(t)=0$, where $k_{\infty}$ is finite.

Proof: From equations (17)-(19) and (20) we have

$$
\dot{e}(t)=c_{1} \widehat{x}_{1}+c_{2} \widehat{x}_{2}-2 u-\dot{y}(t) .
$$

Adding and subtracting $c_{1} \widehat{x}_{2}, c_{1} y(t)$ to the R.H.S. and rearranging we get

$$
\dot{e}(t)=-c_{1} e(t)-2 u+\left(c_{2}-c_{1}\right) \widehat{x}_{2}-c_{1} y(t)-\dot{y}(t) .
$$

By definition $d(t)=\left(c_{2}-c_{1}\right) \widehat{x}_{2}-c_{1} y(t)-\dot{y}(t)$. Temporarily disregarding the term $d(t)$ in (22) and considering the output equation $y_{e}=e(t)$ we get the following system in the error $e(t)$.

$$
\left.\begin{array}{rl}
\dot{e}(t) & =-c_{1} e(t)-2 u \\
y_{e} & =e(t)
\end{array}\right\}
$$

The system in (23), with transfer function $G(s)=$ $-2\left(s+c_{1}\right)^{-1}$ has a minimal representation and is minimum phase. Also for (23), let $A=-c_{1}, B=-2$ and $C=1$, so $\sigma(C B)=-2$. Hence by [18, Theorem 4.2.1] we have; $\lim _{t \rightarrow \infty} k(t)=k_{\infty}$ and $\lim _{t \rightarrow \infty} e(t)=0$. Now we can write eqn. (22) as the system in (23) with some nonlinear perturbations as follows,

$$
\left.\begin{array}{rl}
\dot{e}(t) & =-c_{1} e(t)-2 u+d(t) \\
y_{e} & =e(t)
\end{array}\right\}
$$

From our assumptions $\left(\int_{0}^{\infty}|d(t)|^{p} d t\right)^{\frac{1}{p}}<\infty$, hence $d$ belongs to the space $L_{p}(0, \infty)$. By [18, Theorem 6.1.6] we have that $\lim _{t \rightarrow \infty} k(t)=k_{\infty}$ and $\lim _{t \rightarrow \infty} e(t)=0$. Hence the proof.

Lemma 4.1 allows our simplified system to track the output $y$ of a physical battery as long as the assumptions are met.

\section{B. Battery voltage collapse detection}

Here we formulate a result that allows us to detect terminal voltage collapses for Li-ion batteries by monitoring the states $\widehat{x}_{1}, \widehat{x}_{2}$. First we propose the following algorithm.

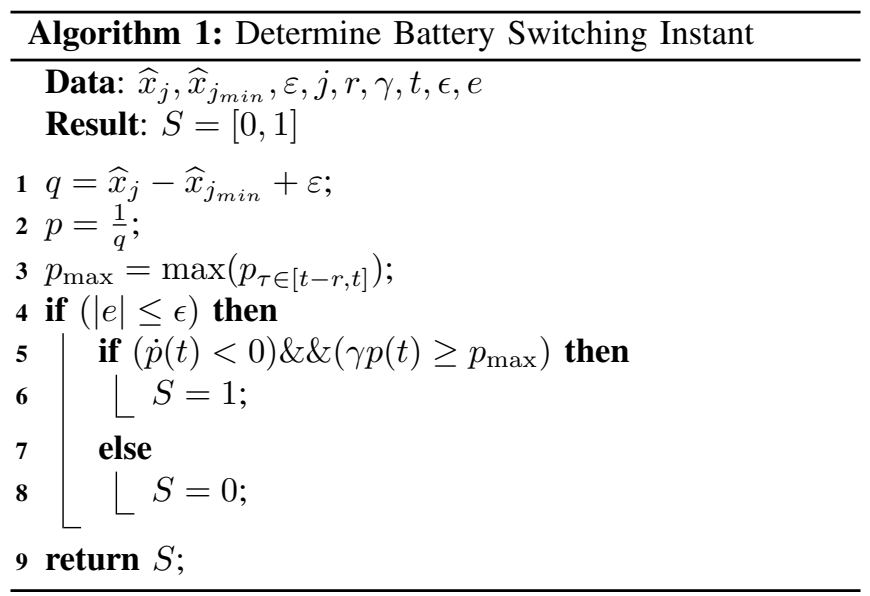

$S$ is an indicator variable in algorithm $1 . S=1$ indicates that the terminal voltage of the Li-ion battery is about to collapse soon. We let $q=\widehat{x}_{j}-\widehat{x}_{j_{\text {min }}}+\varepsilon, p=\frac{1}{q}$, and $j=1$ or 2 . Algorithm 1 monitors the sign of the time derivative of $p$ at time $t$. When the time derivative of $p$ is negative at $t$ and $\gamma p(t) \geq p_{\max }$, then we will prove that $\widehat{x}_{j}$ goes to infinity over time. Here $\gamma>1$ and $p_{\max }$ is the maximum value of $p$ in a time window with length $r$ 
prior to $t$. The condition $|e| \leq \epsilon$ is a guarding condition to ensure that the output of the simplified model $\widehat{y}$ is close enough to the output of the real Li-ion battery $y$. This guard condition ensures that our algorithm is not affected by initial transients in the control effort $u$ due to the UAS strategy and ensures that output $\widetilde{y}$ is sufficiently close to $y$. Inspired by the Razumikhin theorem [22] and its use in [23], [24] we now develop the following result which provides a sufficient condition for detecting terminal voltage collapses for Li-ion batteries.

Theorem 4.2: Assume that the conditions required for Lemma 4.1 to hold are satisfied. Let the discharge current $i$ be bounded, $\gamma>1, q=\widehat{x}_{j}-\widehat{x}_{j_{\text {min }}}+\varepsilon$ and $p=\frac{1}{q}$ where $j=$ 1 or $2, \widehat{x}_{j_{\text {min }}}=\min \left(\widehat{x}_{j}\right)$ and $\varepsilon$ is an infinitesimally small positive constant. Let $p_{\max }=\max _{\tau \in[t-r, t]} p(\tau), 0<r<t$. If $\dot{p}(t) \leq 0$ whenever $\gamma p(t) \geq p_{\max }$ then the physical Li-ion battery is unstable.

Proof: We will prove that the physical Li-ion battery is unstable by contradiction. Let $p_{\max }=\max _{\tau \in[t-r, t]} p(\tau)$ and suppose $\dot{p}(t) \leq 0$ whenever $\gamma p(t) \geq p_{\max }$ but the physical Li-ion battery is stable. Now according to the Razumikhin theorem [25], if $\dot{p}(t)<0$ whenever $\gamma p(t) \geq p_{\max }$ then $p(t)$ converges to zero as $t \rightarrow \infty$. Since $p$ converges to zero we have $q \rightarrow \infty$ with time. But $q=\widehat{x}_{j}-\widehat{x}_{j_{\text {min }}}+\varepsilon$ and $\widehat{x}_{j_{\text {min }}}, \varepsilon$ are constants. This means that $\widehat{x}_{j} \rightarrow \infty$ as $t \rightarrow \infty$. Since $\widehat{x}_{j, j=1}$ or 2 tends to infinity as $t \rightarrow \infty$ we know that either $\widehat{x}_{1}$ or $\widehat{x}_{2}$ goes to infinity as $t \rightarrow \infty$. Now from (19) we have $\widetilde{y}(t) \rightarrow-\infty$ as $t \rightarrow \infty$. But from our assumptions we have that lemma 4.1 holds, i.e. as time tends to infinity, the error $e(t)=\widetilde{y}(t)-y(t) \rightarrow 0$. Hence we have $|y(t)| \rightarrow \infty$ as $t \rightarrow \infty$. Further from equation (11) we have

$$
\begin{aligned}
& |y(t)| \leq\left|E_{0}\right|+\left|-\left(x_{2}+x_{3}+i R_{s}\right)\right| \\
& |y(t)| \leq\left|E_{0}\right|+\left|x_{2}\right|+\left|x_{3}\right|+|i|\left|R_{s}\right|
\end{aligned}
$$

But as per our assumption the physical Li-ion battery described by (8)-(11) is stable. Hence the states $x_{2}, x_{3}$ are bounded for all time. Also the discharge current $i$ is bounded by assumption and $E_{0}, R_{s}$ are bounded by definition. Hence we can say that there exists a real number $M$ such that for all time $t$,

$$
|y(t)| \leq\left|E_{0}\right|+\left|x_{2}\right|+\left|x_{3}\right|+|i|\left|R_{s}\right| \leq M .
$$

This is a contradiction, as above we have established that $|y(t)| \rightarrow \infty$ as $t \rightarrow \infty$. This means our assumption that the physical Li-ion battery is stable, is wrong. Thus by contradiction we have the desired result. This completes the proof.

The above result shows that if $\widehat{x}_{j, j=1}$ or 2 diverges to infinity then the terminal voltage of a Li-ion battery is about to collapse. Thus we have theoretically proven that algorithm 1 can be used to detect that the terminal voltage of a physical battery is about to collapse by monitoring the states $\widehat{x}_{1}$ or $\widehat{x}_{2}$ of the simplified system. Also note that in any real life scenario the discharge current (even in case of a short circuit) is bounded, hence we do not measure it to check it explicitly in our algorithm.

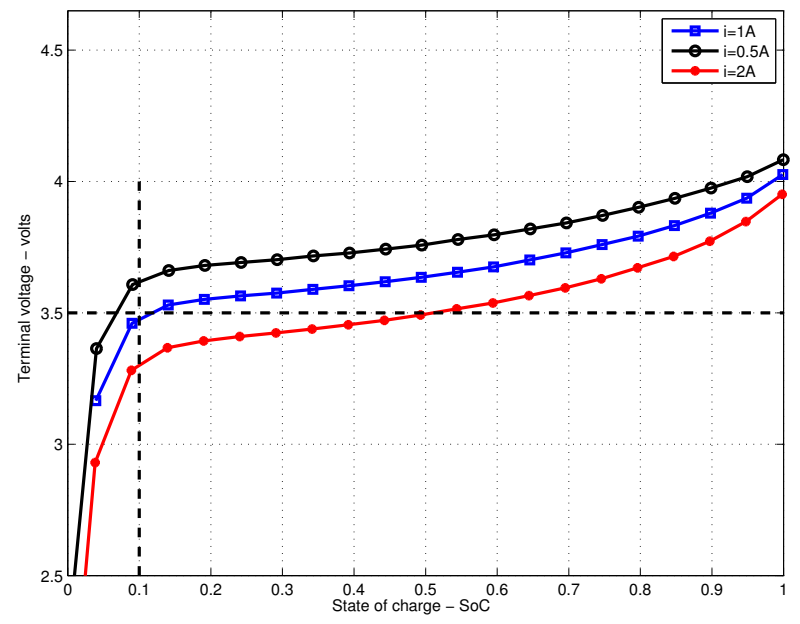

Fig. 4. Terminal voltage vs. SoC for a battery for different discharges. Dashed lines indicate static thresholds.

\section{SIMULATION}

\section{A. Threshold based detection}

First we demonstrate the pitfalls of using simple thresholds to detect an impending terminal voltage collapse. Figure 4 shows the terminal voltage curve vs. SoC for a battery under different discharge currents. The horizontal black dashed line in Fig. 4 represents a terminal voltage threshold of $3.5 \mathrm{~V}$. It detects correctly that the terminal voltage is about to collapse when the load current $i=0.5 A, 1 A$. But when $i=2 A$ using this threshold results in an incorrect detection as the battery still has a $\mathrm{SoC}=50 \%$. Also spikes may appear in the voltage due to sudden large current discharges. This may cause an incorrect detection.

Let us consider preset thresholds on SoC. The vertical black dashed line in Fig. 4 represents a SoC threshold of $10 \%$. For a load of $i=0.5 A$ the battery terminal voltage is above the previously used voltage threshold of $3.5 \mathrm{~V}$ but using the $\mathrm{SoC}$ threshold would remove this battery from service earlier than necessary. Before we show how using our method would help in this situation we present some more simulation results.

\section{B. Comparison of models}

The upper half of fig. 5 shows terminal voltage curves obtained using the CM model in eqns. (8)-(11) and the simplified model in eqns. (13)-(16). Both models are subject to a square wave discharge at $0.25 \mathrm{~Hz}, 1 \mathrm{~A}$ (peak to peak) with a DC offset of $3 \mathrm{~A}$. We assume a battery capacity of $100 \mathrm{mAh}$. The values $2.5,2,1,1,0.5,4.5$ are used for the constants $c_{1}, c_{2}, c_{3}, c_{4}, m$ and $y_{0}$ respectively. Parameters for the CM model can be found in [9]. It is seen from fig. 5 that the voltage curves do not match. The lower half of fig. 5 shows the output of the simplified model in (13)-(16) matches the output of the battery modeled using the $\mathrm{CM}$ model, when the loop is closed as in (20) using the setup shown in fig. 3 . We do not know the values of $c_{1}, c_{2}$ we must pick for the simplified battery model in (13)-(16), but 


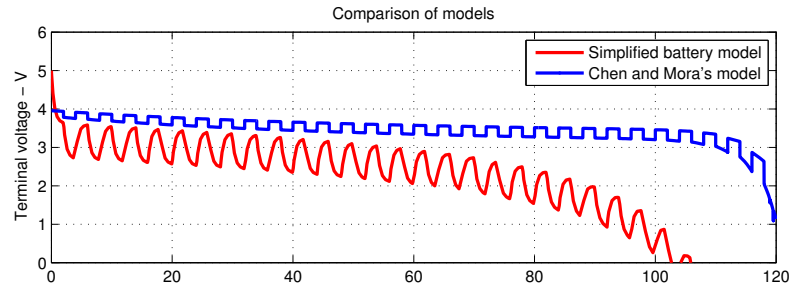

Tracking the battery terminal voltage using UAS

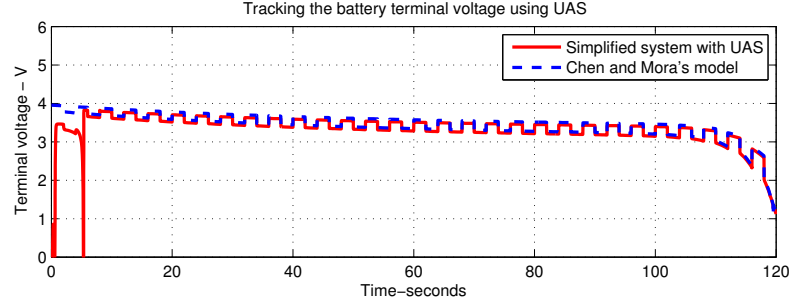

Fig. 5. Comparison of models, performance with and without UAS

we have achieved the desired tracking performance as shown in the lower half of fig. 5. Hence our method works without complete knowledge of the system model.

\section{Detecting terminal voltage collapse using algorithm 1}

For all the following simulations we use a $100 \mathrm{mAh}$ battery, the values of $c_{1}, c_{2}, c_{3}, c_{4}, m$ and $y_{0}$ are the same as used in the previous section and the discharge current always has a magnitude of $1 \mathrm{~A}$ (peak to peak) with a DC offset of $3 \mathrm{~A}$. The output $y$ is produced by running a simulation using the CM model. Figure 6, shows the results of detecting terminal voltage collapse using algorithm 1 for the square wave discharge at $0.25 \mathrm{~Hz}$. The voltage curve for this run is shown in the lower half of fig. 5. The upper half of fig. 6 shows that $\widehat{x}_{1}$ rises as the terminal voltage is about to drop. This is detected at time $t=110 \mathrm{~s}$ when the indicator variable $\mathrm{S}$ in algorithm 1 goes from a zero to a one. At $t=110 \mathrm{~s}$ it is seen from the lower half of fig. 5 that the terminal voltage is just below $3 \mathrm{~V}$ and is beginning to drop quickly. The values $1.001,285,2 \times 10^{-3}$ are used for the variables $\gamma, r, \epsilon$ in algorithm 1 respectively. Note that all window sizes $(r)$ reported here are in terms of number of samples.

Figure 7 shows the results for a sine wave discharge at $0.5 \mathrm{~Hz}$. Algorithm 1 detects an impending voltage collapse at $t=116 \mathrm{~s}$ when $S=1$. At this time the terminal voltage is around $2.5 \mathrm{~V}$ and is seen to be dropping quickly and a sharp rise is seen in the state $\widehat{x}_{1}$. The values $1.001,285,4 \times 10^{-2}$ are used for the variables $\gamma, r, \epsilon$.

Figure 8 shows the results for a square wave discharge at $0.25 \mathrm{~Hz}$ in the presence of Gaussian noise with a covariance of 0.2. Algorithm 1 detects an impending voltage collapse at $t=100 \mathrm{~s}$ when $S=1$. At this time the terminal voltage is around $3.2 \mathrm{~V}$ and is seen to be dropping quickly and a sharp rise is seen in the state $\widehat{x}_{1}$. The values $1.001,277,2 \times 10^{-3}$ are used for the variables $\gamma, r, \epsilon$. From the simulations it appears that tightening the error bound $\epsilon$ helps in the presence of noise or spikes. In general a bigger window size $r$ is seen to perform better but in the presence of noise a
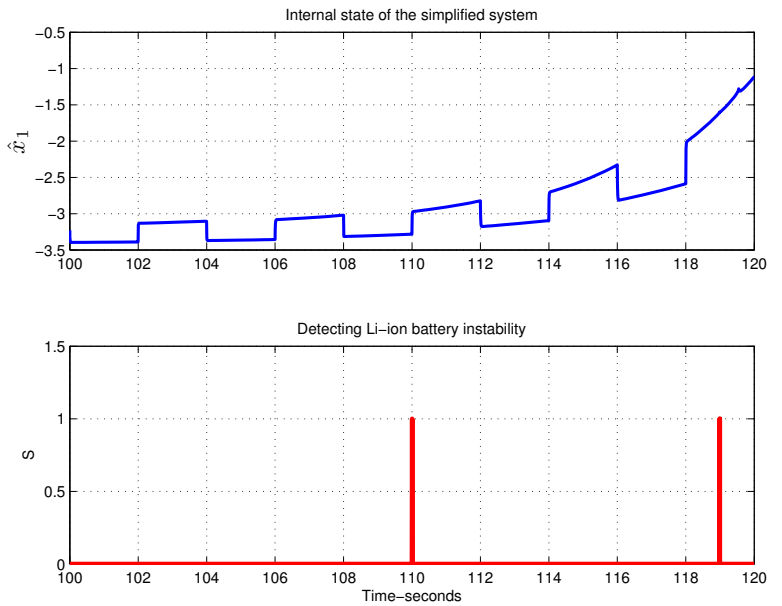

Fig. 6. Rise in $\widehat{x}_{1}$ and the indicator variable $\mathrm{S}$ : square wave discharge
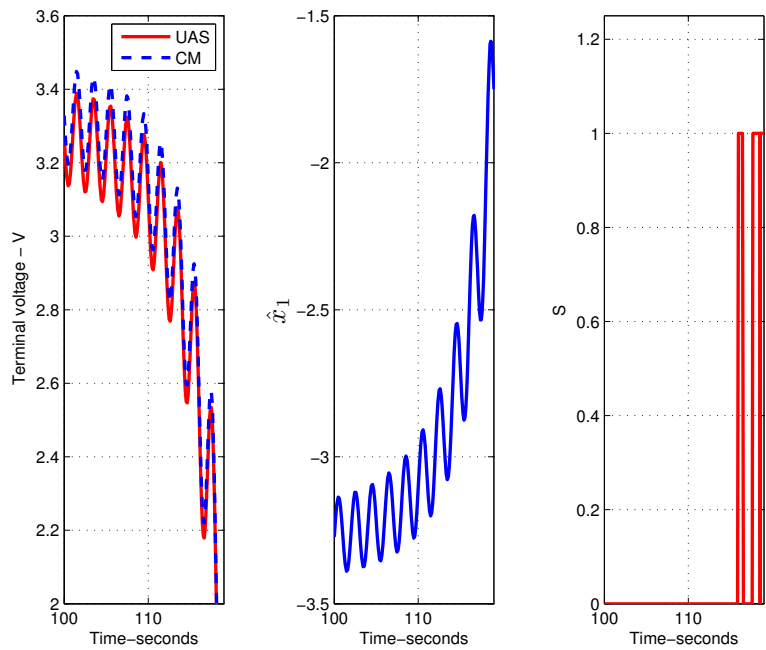

Fig. 7. Falling $y, \widetilde{y}$. Rising $\widehat{x}_{1}$ and the indicator variable $\mathrm{S}$ : sine wave discharge.

slightly smaller window size is helpful.

A typical application using algorithm 1 would be a device powered by a Li-ion battery which can operate as long as the terminal voltage does not fall below $2.5 \mathrm{~V}$. Static thresholds as shown in section $\mathrm{V}$-A can remove a battery from service too early and provide no pre-warning even when they operate correctly. Our algorithm detects impending voltage collapses and hence it can be used for predicting that the terminal voltage will collapse soon. This is the benefit of using our algorithm.

It also appears that algorithm 1 can be used with the terminal voltage $y$ to determine when it is dropping. However then the question to answer is, when should we look at the terminal voltage to determine that it is indeed dropping? If the terminal voltage is fed to algorithm 1 directly, it may report in the very beginning that the voltage is about to collapse. This would be consistent because the terminal voltage of a battery drops linearly over most of its life. Also sharp drops introduced by spikes in the discharge current can 

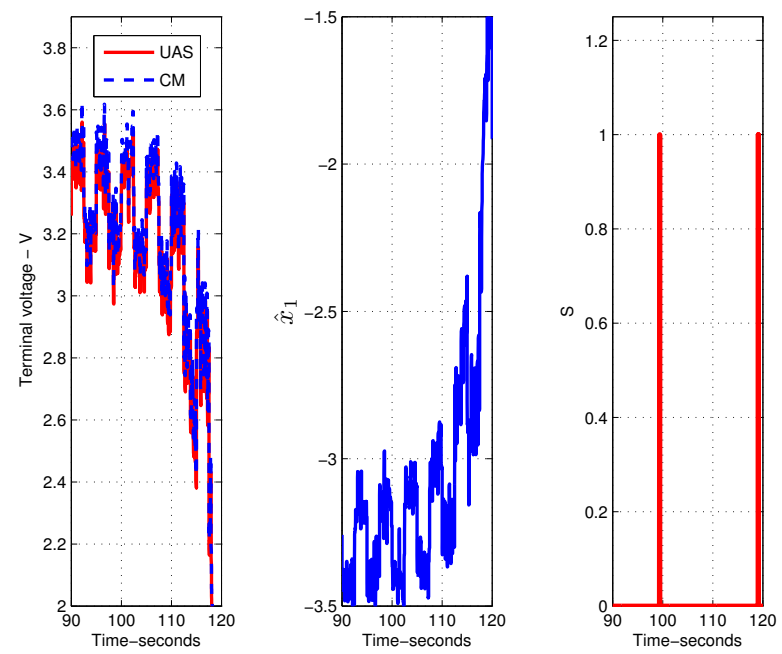

Fig. 8. Falling $y, \widetilde{y}$. Rising $\widehat{x}_{1}$ and the indicator variable S: square wave discharge with measurement noise.

affect the algorithm if used directly with the terminal voltage $y$ as its input. At this point we wish to draw attention to eqns. (11) and (19). From (11) we see that $y$ is affected by changes in the discharge current $i$, where as $\widetilde{y}$ in (19) is not. Even the effects of $u$ on $\widetilde{y}$ are filtered by the dynamics of the simplified system, which is a low pass filter. So intuitively, our method adaptively filters out just enough of the disturbance, but follows the terminal voltage close enough so that essential information about the shape of $y$ vs. $t$ is captured by the states $\widehat{x}_{1}, \widehat{x}_{2}$.

Our method also suggests an unconventional use of the UAS theory, namely, to detect system instability under changing input based on noisy measurement. We believe this approach may be generalizable to other applications.

\section{CONCLUSION}

We have presented a novel method to adaptively detect when the terminal voltage of a Li-ion battery is about to collapse using a simplified battery model. As we do not know the model parameters we use UAS to track the output voltage curve of a physical Li-ion battery. We have mathematically justified our method and demonstrated it using simulations. The method requires measurements of the terminal voltage and does not require measurements of the discharge current. We have demonstrated that our method works in the presence of noise or discharges that are not smooth. Our method is also less susceptible to false alarms which are of concern to static threshold based systems. Since we do not require the details of the actual battery model this method is robust to temperature variations, ageing effects, changes in loading or other nonlinear disturbances.

\section{REFERENCES}

[1] D. Linden and T. B. Reddy, Handbook of Batteries, 3rd ed. McGrawHill, 2002.

[2] R. M. Patil, Z. S. Filipi, and H. K. Fathy, "A Framework for the Integrated Optimization of Charging and Power Management in Plugin Hybrid Electric Vehicles," in American Control Conference, 2012, p. to appear.
[3] T. Wang. and C. G. Cassandras, "Optimal Control of Batteries with Fully and Partially Available Rechargeability," Automatica, p. to appear, 2012.

[4] F. Zhang and Z. Shi, "Optimal and Adaptive Battery Discharge Strategies for Cyber-Physical Systems," in Proceedings of 48th IEEE conference on decision and control, Shanghai, China, 2009, pp. 62326237.

[5] K. B. Hatzell, A. Sharma, and H. K. Fathy, "A Survey of Long-Term Health Modeling, Estimation, and Control Challenges and Opportunities for Lithium-Ion Batteries," in American Control Conference, 2012, p. to appear.

[6] V. Pop, H. Bergveld, D. Danilov, and P. Regtien, Battery Management Systems: Accurate State-of-Charge Indication for Battery Powered Applications. Springer, 2008.

[7] H. Kim and K. G. Shin, "On Dynamic Reconfiguration of a LargeScale Battery System," in RTAS '09: Proceedings of the 2009 15th IEEE Symposium on Real-Time and Embedded Technology and Applications. Washington, DC, USA: IEEE Computer Society, 2009, pp. $87-96$.

[8] M. Coleman, W. G. Hurley, and C. K. Lee, "An Improved Battery Characterization Method Using a Two-Pulse Load Test," IEEE Transactions on Energy Conversion, vol. 23, no. 2, pp. 708-713, Jun. 2008.

[9] M. Chen and R. Mora, "Accurate Electrical Battery Model Capable of Predicting Runtime and I-V Performance," IEEE Transcations on Energy Conversion, vol. 21, no. 2, pp. 504-512, Jun. 2006.

[10] D. Rakhmatov, S. Vrudhula, and D. A. Wallach, "A model for battery lifetime analysis for organizing applications on a pocket computer," IEEE Transactions on VLSI Systems, vol. 11, no. 6, pp. 1019-1030, 2003.

[11] R. Rao, S. Vrudhula, and D. N. Rakhmatov, "Battery modeling for energy-aware system design," Computer, vol. 36 , no. 12 , pp. 77-87, 2003.

[12] M. C. Knauff, C. J. Dafis, D. Niebur, H. G. Kwatny, and C. O. Nwankpa, "Simulink Model for Hybrid Power System Test-bed," in Electric Ship Technologies Symposium, 2007. ESTS '07. IEEE, May 2007, pp. 421-427.

[13] S. Abu-Sharkh and D. Doerffel, "Rapid test and non-linear model characterisation of solid-state Lithium-ion batteries," Journal of Power Sources, vol. 130, no. 1-2, pp. 266-274, 2004.

[14] B. Schweighofer, K. M. Raab, and G. Brasseur, "Modeling of high power automotive batteries by the use of an automated test system," IEEE Transactions on Instrumentation and Measurement, vol. 52, no. 4, pp. 1087-1091, 2003

[15] F. Zhang, Z. Shi, and S. Mukhopadhyay, "Robustness Analysis of Battery Supported Cyber-Physical Systems," ACM Transactions on Embedded Computing Systems, vol. 12, no. 3, pp. in print, arXiv: $1111.5880 \mathrm{v} 1,2012$.

[16] G. L. Plett, "Extended Kalman filtering for battery management systems of LiPB-based HEV battery packs: Part 3. State and parameter estimation," Journal of Power Sources, vol. 134, no. 2, pp. 277-292, 2004.

[17] S. Ding, Model Based Fault Diagnosis Techniques. Springer, 2008.

[18] A. Ilchmann, Non-Identifier Based High Gain Adaptive Control, ser. Lecture Notes in Control and Information Sciences 189, M. Thoma and W. Wyner, Eds. Springer-Verlag, 1993.

[19] S. Mukhopadhyay, Y. Li, and Y. Chen, "Experimental studies of a fractional order universal adaptive stabilizer," in Proceedings of International Conference on Mechtronic and Embedded Systems and Applications, Oct. 2008, pp. $591-596$.

[20] Y. Li, Y. Chen, and Y. Cao, "Fractional Order Universal Adaptive Stabilization," Third IFAC Workshop on Fractional Differentiation and its Applications, Ankara, Turkey, Nov. 2008.

[21] Y. Li and Y. Chen, "When is a Mittag-Leffler function a Nussbaum function?" Automatica, vol. 45, no. 8, pp. 1957-1959, 2009.

[22] S. Zhang and M. Chen, "A new Razumikhin theorem for delay difference equations." Computers and Mathematics with Applications, vol. 36, pp. 10-12, 1998.

[23] W. Wu and F. Zhang, "Robust Cooperative Exploration with a Switching Strategy," IEEE Transactions on Robotics, vol. 28, no. 4, pp. 828839, 2012.

[24] - "A Switching Strategy for Robust Cooperative Exploration," in Proceedings of 49th IEEE Conference on Decision and Control, Atlanta, GA, 2010, pp. 5493-5498.

[25] K. Gu, V. L. Kharitonov, and J. Chen, Stability of Time-Delay Systems. Boston: Birkhäuser, 2003. 